\title{
Barriers to Surgical Resection of Pancreatic Adenocarcinoma
}

\author{
Nicholas Latchana, MD, MSc $\mathrm{c}^{1,2}$ and Natalie Coburn, MD, MPH, FRCSC, FACS ${ }^{1,2,3,4}$ \\ ${ }^{1}$ Department of Surgery, University of Toronto, Toronto, ON, Canada; ${ }^{2}$ Division of General Surgery, Odette Cancer Centre \\ - Sunnybrook Health Sciences Centre, Toronto, ON, Canada; ${ }^{3}$ Sunnybrook Research Institute, Toronto, ON, Canada; \\ ${ }^{4}$ Institute of Clinical Evaluative Sciences, Toronto, ON, Canada
}

We commend Coffman et al. ${ }^{1}$ for examining which groups of patients do not pursue curative-intent resection, and assessing their outcomes. In this analysis of 48,902 patients with resectable pancreatic adenocarcinoma in the National Cancer Data Base, patients who may qualify but do not receive surgical therapy represent an important cohort to enhance our understanding of factors driving patient decision making in complex, high-risk operations, which may afford the only hope for cure. The authors also highlight the threefold improvement in survival for those patients who pursued resection.

Refusal of resection (despite physician recommendation) by $3.7 \%$ of their cohort is higher than reported in other solid-organ malignancies, such as $0.17 \%$ for breast cancer, $0.25 \%$ for colorectal cancer, and $1.5 \%$ for lung cancer. $^{2}$ The magnitude of the operation and the operative risks discussed with the patients are likely major drivers of refusal. The perceived relative oncologic benefit of the operation, and the chance for cure, is weighed against the decision to undergo major operative therapy.

Not surprisingly, age is associated with the refusal of surgery, both in this study and others. This may represent inherent biases among patients and families, as well as providers, who may generalize therapy of pancreas cancer surgery in the elderly as futile. Indeed, we contend that some elderly individuals with poor performance status and a high comorbidity burden should not be considered for surgical resection. However, appropriately selected octogenarians have been shown to tolerate pancreas resection

\footnotetext{
(C) Society of Surgical Oncology 2018
}

First Received: 3 September 2018;

Published Online: 7 November 2018

N. Coburn, MD, MPH, FRCSC, FACS

e-mail: natalie.coburn@sunnybrook.ca without any differences in perioperative morbidity (59 vs. $52 \%, p=0.4)$ and perioperative mortality (3.7 vs. $3.7 \%$, $p=1.0$ ), relative to younger counterparts. ${ }^{3}$ Nonetheless, fear of postoperative complications is more pronounced in the elderly and represents the most common reason for refusal of treatment in patients. ${ }^{2,4}$ For a subgroup of patients refusing surgery, Coffmann et al. ${ }^{1}$ show that chemoradiation may offer improved survival compared with no resection, and this may indeed be a preferred route for some patients.

More troubling are the findings in this study and others that race, lower annual income, and marital status are associated with the decision to pursue curative-intent surgery. Married individuals are diagnosed earlier and benefit from improved overall survival (after controlling for stage at diagnosis and treatment), ${ }^{5}$ with the association between marital status and improved cancer survival likely related to higher socioeconomic status and better social support. ${ }^{5}$ Racial disparities for pancreas cancer are previously reported, with African Americans experiencing higher ageadjusted incidence and worse prognosis relative to Caucasians. ${ }^{6-8}$ Access to healthcare services is critical as certain populations remain underinsured and are less likely to be treated at centers of excellence. ${ }^{8}$ Often presenting with a higher burden of comorbidities and more advanced disease, it is not surprising that African Americans are less likely to undergo surgical resection and receive chemotherapy and radiation; however, this alone does not entirely account for disparities in overall survival. ${ }^{8}$ Beyond access to care, racial disparities appear to persist among those being considered for surgical resection, as identified in this study (despite similarities in resectability between races). ${ }^{9}$ Importantly, once resection is performed, race is no longer a significant predictor of survival. ${ }^{6}$ Understanding and addressing modifiable barriers that limit access to resection for African Americans remains warranted. 
Gender disparity is evident among those undergoing surgical intervention across genders in some neoplastic settings. ${ }^{10}$ Shugarman et al. ${ }^{11}$ found women were $25 \%$ less likely than men to receive surgery within 6 weeks of diagnosis for non-small cell lung cancer. By report, women are more afraid of surgery and may avoid surgical intervention to prevent disruption of their caregiver roles. ${ }^{12}$ Citrin et al. ${ }^{13}$ found women who refuse breast cancer treatment may be more likely to accept treatment if physicians acknowledged patient fears, provided hope, described treatment possibilities, and provided time to cope with the diagnosis prior to starting treatment. ${ }^{13}$

A broader problem is obtaining access to health services among those who are uninsured, underinsured, and lacking the socioeconomic supports needed to pursue complex cancer therapies. Despite overcoming the initial barrier of access into healthcare systems for those within the study, insurance status was still associated with the ability to pursue surgical resection. Importantly, the true number of uninsured patients who do not receive surgery for pancreas adenocarcinoma may be underrepresented in this study as the National Cancer Data Base only captures patients who have received services at an American College of Surgeons Commission on Cancer-accredited facility. ${ }^{14}$ Even for insured individuals, variation in deductibles and coverage, inability to travel for care or take time off work could be financially prohibitive, and contribute to their refusal of surgery. In Canada, with its universal healthcare system, survival for pancreas adenocarcinoma was associated with similar factors identified in this study, including age, gender, and comorbidities, as well as other factors such as urban residence with lower income, rural residence, resident instability, and material deprivation. ${ }^{15}$ Other universal health systems, such as in Denmark, have also found similar factors associated with pancreas cancer survival, e.g. urban living. ${ }^{16}$ This suggests that insurance status alone does not account for the differences in surgical intervention, and more support may need to be given to patients of lower socioeconomic status to assist in the navigation of complex treatments.

Given the marked difference in survival between those undergoing resection and those who refuse surgery, further examinations of patient preferences, and the optimal ways to support patients through this complex journey, are necessary.

DISCLOSURES Nicholas Latchana and Natalie Coburn have no disclosures to declare.

\section{REFERENCES}

1. Coffman A, Torgeson A, Lloyd S. Correlates of refusal of surgery in the treatment of non-metastatic pancreatic adenocarcinoma. Ann Surg Oncol. 2018. https://doi.org/10.1245/s10434-018-6708-y.

2. Aizer AA, Chen MH, Parekh A, et al. Refusal of curative radiation therapy and surgery among patients with cancer. Int $J$ Radiat Oncol Biol Phys. 2014;89(4):756-64.

3. Hatzaras I, Schmidt C, Klemanski D, et al. Pancreatic resection in the octogenarian: a safe option for pancreatic malignancy. $J \mathrm{Am}$ Coll Surg. 2011;212(3):373-7.

4. Rothman MD, Van Ness PH, O'Leary JR, Fried TR. Refusal of medical and surgical interventions by older persons with advanced chronic disease. J Gen Intern Med. 2007;22(7):982-7.

5. Goodwin JS, Hunt WC, Key CR, Samet JM. The effect of marital status on stage, treatment, and survival of cancer patients. JAMA. 1987;258(21):3125-30.

6. Murphy MM, Simons JP, Hill JS, et al. Pancreatic resection: a key component to reducing racial disparities in pancreatic adenocarcinoma. Cancer. 2009;115(17):3979-90.

7. Wray CJ, Castro-Echeverry E, Silberfein EJ, Ko TC, Kao LS. A multi-institutional study of pancreatic cancer in Harris County, Texas: race predicts treatment and survival. Ann Surg Oncol. 2012;19(9):2776-81.

8. Khawja SN, Mohammed S, Silberfein EJ, Musher BL, Fisher WE, Van Buren G. Pancreatic cancer disparities in African Americans. Pancreas. 2015;44(4):522-7.

9. Abraham A, Al-Refaie WB, Parsons HM, Dudeja V, Vickers SM, Habermann EB. Disparities in pancreas cancer care. Ann Surg Oncol. 2013;20(6):2078-87.

10. Sjöström C, Thorstenson A, Ströck V, et al. Treatment according to guidelines may bridge the gender gap in outcome for patients with stage T1 urinary bladder cancer. Scand J Urol. 2018. https://doi.org/10.1080/21681805.2018.1462254.

11. Shugarman LR, Mack K, Sorbero ME, et al. Race and sex differences in the receipt of timely and appropriate lung cancer treatment. Med Care. 2009;47(7):774-81.

12. Karlson EW, Daltroy LH, Liang MH, Eaton HE, Katz JN. Gender differences in patient preferences may underlie differential utilization of elective surgery. Am J Med. 1997;102(6):524-30.

13. Citrin DL, Bloom DL, Grutsch JF, Mortensen SJ, Lis CG. Beliefs and perceptions of women with newly diagnosed breast cancer who refused conventional treatment in favor of alternative therapies. Oncologist. 2012;17(5):607-12.

14. Boffa DJ, Rosen JE, Mallin K, et al. Using the National Cancer Database for outcomes research: a review. JAMA Oncol. 2017;3(12):1722-8.

15. Kagedan DJ, Abraham L, Goyert N, et al. Beyond the dollar: influence of sociodemographic marginalization on surgical resection, adjuvant therapy, and survival in patients with pancreatic cancer. Cancer. 2016;122(20):3175-82.

16. Kirkegård J, Ladekarl M, Fristrup CW, Hansen CP, Sall M, Mortensen FV. Urban versus rural residency and pancreatic cancer survival: a Danish nationwide population-based cohort study. PLoS ONE. 2018;13(8):e0202486. 